\title{
Male-Specific Submaxillary Gland Protein, a Lipocalin Allergen of the Golden Hamster, Differs from the Lipocalin Allergens of Siberian and Roborovski Dwarf Hamsters
}

\author{
Christiane Hilger $^{\mathrm{a}}$ Ved P. Dubey ${ }^{\mathrm{f}}$ Delphine Lentz ${ }^{\mathrm{a}} \quad$ Caroline Davril $^{\mathrm{a}}$ \\ Dominique Revets $^{\mathrm{b}} \quad$ Claude $P$. Muller $^{\mathrm{b}} \quad$ Claire Diederich $^{\mathrm{e}}$ Hélène De La Barrière ${ }^{c}$ \\ Françoise Codreanu-Morel $^{d}$ Martine Morisset ${ }^{d} \quad$ Christiane Lehners $^{d}$ Prabir K. De ${ }^{f}$ \\ François Hentges ${ }^{a}$

\begin{abstract}
Departments of a Infection and Immunity and ${ }^{\mathrm{b}}$ Immunology, Luxembourg Institute of Health, ' Unit of Pneumology and ${ }^{\mathrm{d}}$ National Unit of Immunology-Allergology, Centre Hospitalier de Luxembourg, Luxembourg, Luxembourg; ${ }^{\text {eDepartment }}$ of Veterinary Medicine, University of Namur, Namur, Belgium; ${ }^{f}$ CSIR-Centre for Cellular and Molecular Biology, Hyderabad,
\end{abstract} \\ India
}

\section{Key Words}

Allergic asthma · Golden hamster allergen - Lipocalin .

Salivary gland $\cdot$ Male-specific submaxillary gland protein

\begin{abstract}
Background: An increasing number of asthma cases upon exposure to hamsters and anaphylactic reactions following hamster bites are being reported, but the allergens responsible are still poorly characterized. In the Golden hamster, male-specific submaxillary gland protein (MSP), a lipocalin expressed in a sex- and tissue-specific manner in the submaxillary and lacrimal glands, is secreted in the saliva, tears and urine. The purpose of this study was to determine if MSP is an allergen, to identify lgE-reactive proteins of different hamster species and to analyse potential cross-reactivities. Methods: Fur extracts were prepared from four hamster species. Hamster-allergic patients were selected based on a history of positive lgE-test to hamster epithelium. The lgE-reactivity of patients' sera was investigated by means of immunoblot and ELISA. IgE-reactive proteins in fur extracts and
\end{abstract}

(c) 2015 S. Karger AG Basel

1018-2438/15/1661-0030\$39.50/0 the submaxillary gland were identified using anti-MSP antibodies, Edman sequencing or mass spectrometry. MSP was purified from Golden hamster and recombinant MSP was expressed in E. coli. Results: Four patients had IgE-antibodies against 20.5-kDa and 24-kDa proteins of Golden hamster fur extract, which were identified as MSP. IgE-reactive MSP-like proteins were detected in European hamster fur extract. Three patient sera showed IgE-reactive bands at 17-21 kDa in Siberian and Roborovski hamster fur extracts. These proteins were identified as two closely related lipocalins. Immunoblot inhibition experiments showed that they are crossreactive and are different from MSP. Conclusion: MSP lipocalin of the Golden hamster was identified as an allergen, and it is different from the cross-reactive lipocalin allergens of Siberian and Roborovski hamsters. Our findings highlight the need for specific tools for the in vitro and in vivo diagnosis of allergy to different hamster species.

Correspondence to: Dr. Christiane Hilger

Department of Infection and Immunity, LIH

84 Val Fleuri

LU-1526 Luxembourg (Luxembourg)

E-Mail christiane.hilger@lih.lu

Correspondence to: Dr. Prabir Kumar De

CSIR-Centre for Cellular and Molecular Biology

Uppal Road

Hyderabad, 500007 (India)

E-Mail pkde@ccmb.res.in 


\section{Introduction}

Apart from cats and dogs, which represent the most frequent animals to be kept as pets in industrialized countries, a number of other mammalian furry pets have become increasingly popular. They are called 'exotic pets' or 'new companion animals' and include rodents (hamsters, guinea-pigs, chinchillas, gerbils, mice and rats), mustelids (ferrets), leporids (rabbits) or even miniature pigs. In parallel to the emergence of these new pets, clinical cases were published in the literature reporting on allergic symptoms caused by these animals [1]. It is difficult to evaluate the incidence of these allergies. Indeed, the prevalence of exotic pets in households is not well known, the molecules implicated in allergic sensitization are mostly not characterized and purified allergen components are not commercially available for diagnosis. The use of animal hair or epithelial extracts does not allow for distinguishing reliably between different animals due to crossreactive molecules which generate false-positive results. On the other hand, poor standardization of extracts can give false-negative results.

The three most popular pet hamster species are the Golden or Syrian hamster (Mesocricetus auratus) and the two dwarf hamsters, the Siberian or Djungarian hamster (Phodopus sungorus) and the Roborovski hamster (Phodopus roborovskii). The European, common or blackbellied hamster (Cricetus cricetus) is less popular as a pet. The Golden hamster is the main species used in animal research experiments. A number of studies have reported bronchial asthma upon exposure to hamsters as well as the occurrence of anaphylactic reactions after hamster bites [2-10]. Most cases are related to Siberian hamsters; only 1 study reported the occurrence of symptoms with other hamster species, namely the Roborovski, Golden and European hamster [4]. It appears, however, that the allergens originating from these four species are not identical. Indeed, patients allergic to the Siberian hamster did not react to commercial skin-prick test (SPT) preparations made from the European or the Golden hamster [2, $3,5,7,8,10]$.

A recent report of anaphylaxis induced by the bite of a Siberian hamster [7] described an allergen in the fur, salivary glands and urine, which seems to exist as 3 isoforms of 18,21 and $23 \mathrm{kDa}$. So far, no allergens have been described for the Golden or Roborovski hamsters. In the latter, a lipocalin called roborovskin has been identified in the urine [11], but its distribution in the body fur, its tissue of origin and whether it might be an allergen have not been investigated. In the Golden hamster, a male-spe-

Golden Hamster Allergen MSP Differs

from Dwarf Hamster Lipocalin Allergens cific submaxillary gland protein (MSP) has been identified, which is secreted in the saliva and excreted in the urine of males. MSP, which exists in glycosylated as well as non-glycosylated forms, is a well-characterized lipocalin, having the closest identity with the odorant-binding lipocalins [12-15]. Low levels of MSP are also expressed female-specifically in the lacrimal glands and secreted in the tears of females [14]. The presence of MSP in the body fur of Golden hamsters and whether it is an allergen have not been investigated.

Most allergenic proteins of animal origin are members of the lipocalin family, sharing low-to-modest sequence identities but with a highly conserved tridimensional structure [16]. The aim of this work was to study whether the lipocalin MSP is implicated in allergy to the Golden hamster and to identify IgE-reactive allergens in Siberian and Roborovski dwarf hamsters and analyse potential cross-reactivities.

\section{Material and Methods}

\section{Patients and Sera}

Patients were selected from the outpatient population of the National Unit of Immunology and Allergology and the Unit of Pneumology of the Centre Hospitalier in Luxembourg, based on a history of specific IgE to hamster epithelium e84 (ImmunoCAP, Thermo Fisher Scientific, Uppsala, Sweden). The source material for the ImmunoCAP is a mixture of epithelia from 3 hamsters: European, Golden and Siberian. Six sera were found to be positive and 4 patients could be re-contacted for further clinical assessment and SPT. The study was approved by the National Committee for Medical Research Ethics.

Patient 1 (P1) had kept a male hamster of unknown species for 2 years, and she had asthma and rhinoconjunctivitis upon contact with it (table 1). Patient 2 (P2) had two female dwarf hamsters of unknown species for 1 and 2 years, respectively. She also had asth$\mathrm{ma}$ and rhinoconjunctivitis upon contact with her hamsters. Patient 3 (P3) was lost from follow-up. Patient 4 (P4) experienced wheezing, conjunctival pruritus, labial and palpebral oedema a few minutes after kissing her Golden hamster, and subsequently presented with pruritus, rhinitis and allergic conjunctivitis upon contact with her pet. Patient 5 (P5) had asthma and rhinitis upon contact with his hamster; the symptoms started about 10 months after the acquisition of the pet. Patient 6 (P6) had perennial asthma and rhinoconjunctivitis. He had a cat at home and had had a hamster of unknown species in childhood (at 10-14 years of age), but without presenting any clearly hamster-related allergic symptoms. At the time of follow-up for SPT and additional investigations, none of the 4 patients had a hamster at home.

Sera from 16 cat- and/or dog-allergic and 10 guinea-pig-allergic patients were collected for control purposes. The selection was based on the presence of specific IgE to cat dander el or dog dander e5 (ImmunoCAP) in the case of cat/dog-allergic patients (range 1.0 to $>100 \mathrm{kUA} / \mathrm{l}$ to cat dander, mean 21.1 ; range 0.4 to $29.5 \mathrm{kUA} / \mathrm{l}$ to dog dander, mean 6.1). The sera of guinea-pig-allergic patients 
Table 1. Patient data

\begin{tabular}{|c|c|c|c|c|c|c|}
\hline & $\mathrm{P} 1$ & $\mathrm{P} 2$ & $\mathrm{P} 3$ & $\mathrm{P} 4$ & P5 & P6 \\
\hline Sex, male/female; age, years & female; 42 & female; 44 & male; 52 & female; 51 & male; 23 & male; 36 \\
\hline Pet hamster & male, unknown species & 2 female dwarf species & n.d. & $\begin{array}{l}\text { Golden hamster } \\
\text { of unknown sex }\end{array}$ & unknown & unknown \\
\hline IgE hamster epithelium, kUA/l & 5.6 & 11.2 & 16 & 0.45 & 4.6 & 17 \\
\hline \multicolumn{7}{|l|}{ SPT, mm } \\
\hline $\begin{array}{l}\text { Golden hamster } \\
\text { (Stallergenes) }\end{array}$ & $6 \times 5(1,800 \mu \mathrm{g} / \mathrm{ml})$ & $5 \times 4$ & n.t. & $5.5 \times 6$ & negative & $25 \times 15$ \\
\hline European hamster (ALK) & $8 \times 6(300 \mu \mathrm{g} / \mathrm{ml})$ & $4 \times 3.5$ & n.t. & $7 \times 4.5$ & n.t. & $8 \times 12$ \\
\hline
\end{tabular}

n.d. $=$ No data; n.t. $=$ not tested .

were selected on the basis of positive specific IgE to guinea-pig epithelium e6 (ImmunoCAP; range 5.9-41 kUA/l, mean 18.1). Twenty-two additional sera were collected from mite and/or pollen-allergic patients with no history of allergy to furred animals.

\section{Protein Extractions}

The body fur of male Golden hamsters (bred and maintained at the Centre for Cellular and Molecular Biology animal house) was shaved using an electric razor, stirred with autoclaved Milli-Q water for $1 \mathrm{~h}$ and the extract was collected after centrifugation. The submaxillary gland (SMG) extracts of male Golden hamsters were prepared by homogenization $(2.5 \% \mathrm{w} / \mathrm{v})$ in chilled $20 \mathrm{mM}$ Tris$\mathrm{HCl}(\mathrm{pH} 7.4)$ and centrifugation at $28,000 \mathrm{~g}$ for $30 \mathrm{~min}$ at $4{ }^{\circ} \mathrm{C}$ as described $[12,15]$. Golden hamsters were handled according to the Centre's Institutional Animal Experimentation Ethics guidelines.

Adult male (Siberian and Roborovski) dwarf hamsters were obtained from a local breeder. Their fur was cut with scissors and the proteins were extracted under rotation overnight with phosphatebuffered saline (PBS) containing protease inhibitors (protease inhibitor cocktail tablets, Roche, Mannheim; Germany). The SMGs were harvested immediately after sacrifice and frozen at $-20^{\circ} \mathrm{C}$. They were disrupted with beads (TissueLyser II, Qiagen, Hilden, Germany) and extracted in buffer containing $50 \mathrm{mM}$ Tris $\mathrm{HCl}(\mathrm{pH}$ $8.0), 150 \mathrm{mM} \mathrm{NaCl}$ and protease inhibitors. The animals were handled according to the European guidelines for experimental animals. European hamster hair and skin scrape were obtained from Allergon (Ängelholm, Sweden).

\section{Production of Native and Recombinant MSP}

Natural MSP (nMSP) lipocalins present in adult male Golden hamster SMG extracts (as $24-\mathrm{kDa}, 20.5-\mathrm{kDa}$ and $30-\mathrm{kDa}$ forms, which differ in the presence or absence of N-glycosylation [13-
15]) were purified employing a rapid procedure. The proteins precipitated from the extract within an $85-100 \%\left(\mathrm{NH}_{4}\right)_{2} \mathrm{SO}_{4}$ saturation were collected by centrifugation, dissolved in $20 \mathrm{mM}$ Tris- $\mathrm{HCl}$ containing $150 \mathrm{mM} \mathrm{NaCl}$ ( $\mathrm{pH} 7.4$ ) and resolved in a gel-filtration column $(1.6 \times 95 \mathrm{~cm}$; Sephadex-G75 superfine; GE Healthcare, Buckinghamshire, UK) equilibrated with the same buffer. Eluted fractions were checked in SDS-PAGE and if required, by Western blot using anti-MSP antibodies. Selected fractions of the most prominent and broad protein peak of gel-filtration, in which the 3 MSP forms elute (with considerable overlap but free of other proteins) were pooled. The final pool contained 24-kDa MSP (N-glycosylated) and $20.5-\mathrm{kDa}$ MSP (non-glycosylated) as the major components, along with low levels of a 30-kDa, higher N-glycosylated form of MSP.

The cDNA encoding the 157 aa long, mature polypeptide of MSP was amplified by RT-PCR using total RNA isolated from the SMG of the male Golden hamster and specific primers based on the known cDNA sequence of MSP (AF183407.2) [13, 14]. The amplified cDNA of the mature MSP was cloned in pET21a vector at Nde1/Xho1 sites, such that the sequence of the encoded recombinant MSP (rMSP) included a C-terminal 6X His-tag. BL21 (DE3) Escherichia coli cells transformed with the above plasmid construct were cultured, and the expression of rMSP (induced by the addition of isopropyl $\beta$-D-1-thiogalactopyranoside) and its purification from the cell lysate were performed exactly as described earlier [17]. Pure rMSP eluted as a monomer in a single peak (at approx. $20 \mathrm{kDa}$ ) in the final gel-filtration step.

\section{SDS-PAGE, IgE-Immunoblotting and IgE-Inhibition}

Proteins were separated by using 15\% SDS-PAGE under reducing conditions and transferred to PVDF membranes (Millipore, Bedford, Mass., USA). Briefly, patient sera were diluted 1:3 or 1:6 
in blocking buffer (Tris-buffered saline/0.05\% Tween 20 and 3\% bovine serum albumin; Sigma-Aldrich, Diegem, Belgium). Proteins blotted on the membrane were incubated overnight at room temperature with the diluted patient sera. Bound IgE-antibodies were detected with anti-human IgE-antibodies labelled with alkaline phosphatase (Southern Biotech, Birmingham, Ala., USA). Specific bands were visualized by colour development using nitroblue tetrazolium/5-bromo-4-chloro-3-indolyl-phosphate (NBT/ BCIP) (Promega, Leiden, The Netherlands). MSP was detected using polyclonal rabbit antibodies (IgG), which were purified by protein-A sepharose affinity from the crude anti-serum raised earlier against pure $\mathrm{N}$-glycosylated $24 \mathrm{kDa}$ natural MSP [13]. An antirabbit IgG antibody labelled with alkaline phosphatase (Sigma-Aldrich) was used as a secondary antibody for detection. Two-dimensional (2D)-gel electrophoresis was performed with immobilized $\mathrm{pH}$ gradient strips according to the manufacturer's instructions (Bio-Rad, Nazareth, Belgium). The proteins were checked for the presence of glycosylation using a commercial kit (glycoprotein staining kit, Pierce, Erembodegem, Belgium).

For IgE inhibition assays, patients' sera were diluted and incubated with $20 \mu \mathrm{g}$ of different extracts or $10 \mu \mathrm{g}$ of purified nMSP for $2 \mathrm{~h}$ at room temperature before being added to the membrane strips for the detection of IgE-binding proteins.

Detection of Specific IgE Antibodies by ELISA

Specific IgE to native MSP, recombinant MSP and hamster albumin (Sigma) were quantified by ELISA. Briefly, the proteins were coated overnight at a concentration of $5 \mu \mathrm{g} / \mathrm{ml}$ in PBS to microtitre plates (NUNC MaxiSorp ${ }^{\mathrm{TM}}$, Rosklide, Denmark). Unspecific binding sites were blocked by the addition of blocking buffer (3\% BSA in PBS) for 30 min. Patient sera were diluted 1:3 or 1:6 in blocking buffer and added to the wells for $2 \mathrm{~h}$. Between each step, the plates were washed with washing buffer (Tris-buffered saline $/ 0.05 \%$ Tween-20). The binding of specific IgE was measured by addition of biotin-labeled anti-human IgE (Southern Biotech) for $1 \mathrm{~h}$, followed by alkaline-phosphatase-labeled streptavidin (BD Biosciences, Erembodegem, Belgium) for $30 \mathrm{~min}$. Plates were developed by addition of para-nitrophenyl phosphate (p-NPP) (Sig$\mathrm{ma}$ ) and optical density was read at $405 \mathrm{~nm}$. A series of 22 sera from patients allergic to mites and/or pollen were used as negative controls. The quantification of specific IgE to hamster fur extracts was performed as described above, except that $8 \mu \mathrm{g}$ of protein $/ \mathrm{ml}$ were coated to the microtitre plate.

\section{SPT with Extracts and Native Allergens}

Commercial SPT solutions were used from the following suppliers: ALK, Horsholm, Denmark containing C. cricetus (European hamster) and Stallergenes, Antony, France, containing M. auratus (Golden hamster) hair extracts. The protein concentrations of the commercial SPT solutions were quantified by Bradford assay (Bio-Rad). Hair (fur) extracts from the two dwarf hamsters $P$. sungorus (Siberian hamster) and P. roborovskii (Roborovski hamster) prepared in the laboratory were sterilized by filtration and diluted in human serum albumin diluent (ALK) with the following concentrations of $0.1,1,10$ and $50 \mu \mathrm{g} / \mathrm{ml}$. nMSP was diluted in the diluent to $0.1,1$ and $10 \mu \mathrm{g} / \mathrm{ml}$. The diluent was used as negative control, histamine chloride $(10 \mathrm{mg} / \mathrm{ml})$ was used as a positive control and skin reactions were read after 20 min. Prick tests yielding a papule $\geq 3 \mathrm{~mm}$ above the negative control were rated positive.

Golden Hamster Allergen MSP Differs from Dwarf Hamster Lipocalin Allergens
Protein Sequencing and Mass Spectrometry

For N-terminal sequencing, proteins were separated by SDSPAGE and transferred onto PVDF membranes by semi-dry transfer using CAPS-buffer [3-(cyclohexylamino)-1-propanesulfonic acid, CAPS $10 \mathrm{mM}$, methanol 20\%]. Purified proteins were subjected to N-terminal sequencing by automated Edman degradation on a PROCISE 49X HT protein sequencer (Applied Biosystems, Foster City, Calif., USA). For analysis by mass spectrometry, IgE-reactive spots were cut out from 2D-gels stained with Gelcode Blue (Pierce, Erembodegem, Belgium).

For mass spectrometry analyses, the isolated spots were digested in gel with trypsin (sequencing grade, Roche). Digested peptides were analysed as described [18] on a MALDI TOF/TOF instrument (Ultraflex I, Bruker Daltonics, Bremen, Germany). Proteins were identified by comparison of the mass lists of their peptide mass fingerprints with the NCBInr database and the in silico digestion of the corresponding sequences (peptide mass, Expasy, Swiss Institute of Bioinformatics, Lausanne, Switzerland). Some unknown digested peptides were de novo sequenced on a second MALDI TOF/TOF instrument (AB Sciex 5800, Framingham, Mass., USA) to extend the sequence coverage and improve the characterization of the isoforms.

\section{Results}

\section{IgE-Reactive Proteins in Fur Extracts from Four Hamster Species}

The aqueous body-fur/hair extracts of four hamster species (Golden, European, Siberian and Roborovski) were separated by SDS-PAGE and immunoblotted with sera from 6 patients (fig. 1) who had been found positive to hamster epithelium (e84) with ImmunoCAP. Sera from P2, P3, P4 and P6 but not from P1 and P5 detected IgE-reactive proteins at approx. $20 \mathrm{kDa}$ and $24 \mathrm{kDa}$ in Golden hamster fur (fig. 1a). Similar bands were visible in the European hamster fur extract with sera from P3 and P4 (fig. 1b).

In the Siberian hamster fur extract, the sera from P1, $\mathrm{P} 2$ and P5 detected major IgE-reactive bands in the 17- to 21-kDa region (fig. 1c). These IgE-reactive bands are very similar to those described by Torres et al. [7] in Siberian hamster fur extract. The sera of the same 3 patients also detected an IgE-reactive band in Roborovski hamster fur at $21 \mathrm{kDa}$ (fig. 1d) as well as low-molecular-weight bands of about $6 \mathrm{kDa}$ in the Siberian and Roborovski hamster fur extracts. The serum of $\mathrm{P} 2$ was strongly reactive to Siberian and Roborovski hamster fur, but it also showed reactivity to Golden hamster fur.

The fact that $\mathrm{P} 3$ and $\mathrm{P} 4$ reacted only to the Golden and European hamsters in the IgE immunoblots, but not to the two dwarf hamster species, suggests that the allergens of the Golden and European hamsters might be different from those of the Siberian or Roborovski hamsters.

Int Arch Allergy Immunol 2015;166:30-40 
Fig. 1. IgE-reactive proteins in fur extracts from four hamster species. a-d Proteins were separated by SDS-PAGE under denaturing conditions and analysed on IgE immunoblot using sera from 6 patients allergic to hamsters. MSP was detected by polyclonal rabbit antibodies. Numbered lanes correspond to the sera of P1-P6. N = Negative control; $\mathrm{R}=$ rabbit anti-MSP antibody.

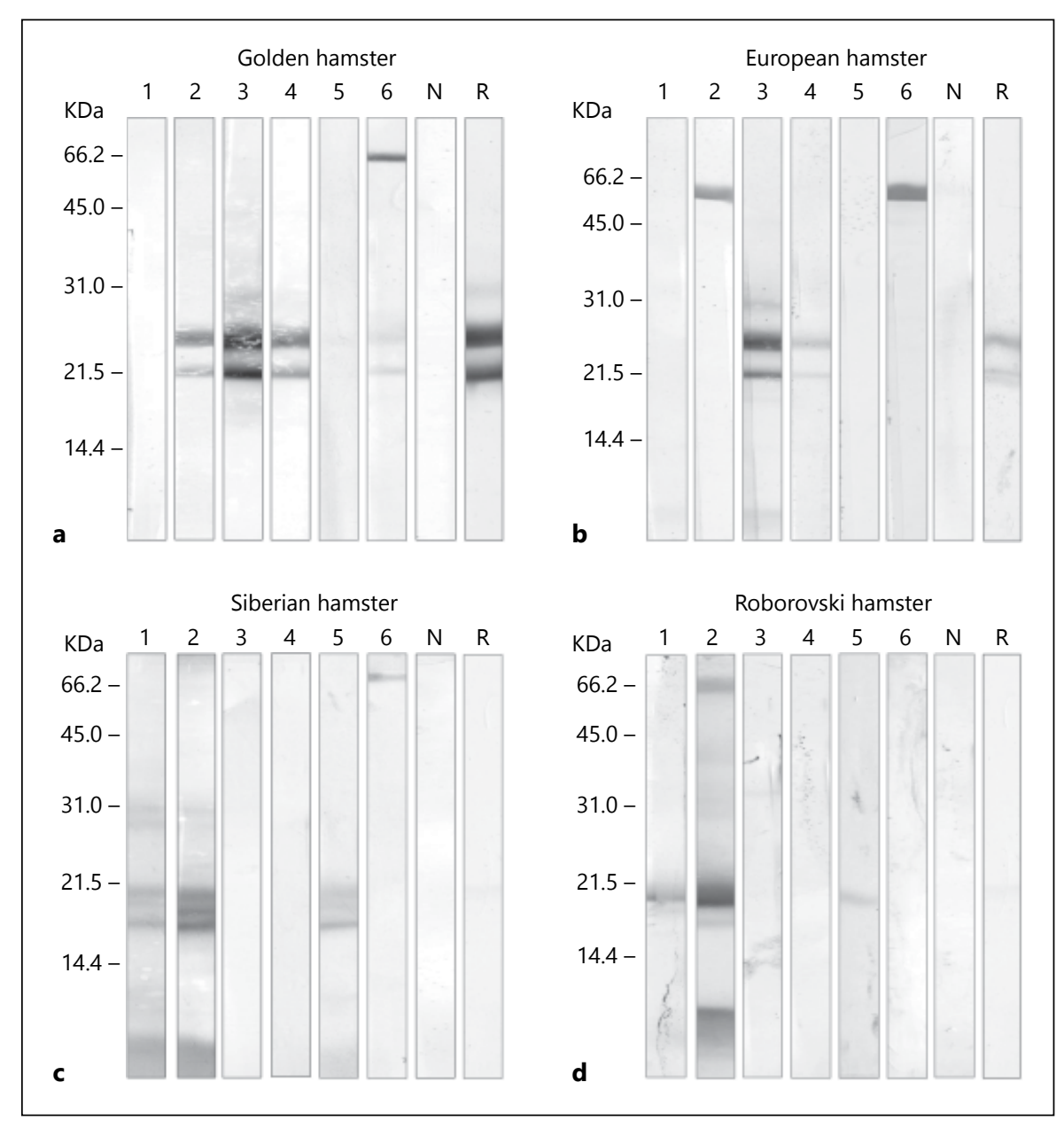

Detection of MSP by Rabbit Anti-MSP Antibodies

MSP lipocalin is known to be present in the SMG, saliva and urine of male Golden hamsters as the $\mathrm{N}$-glycosylated $24-\mathrm{kDa}$ and non-glycosylated $20.5-\mathrm{kDa}$ major forms, along with a minor, higher $\mathrm{N}$-glycosylated form of approximately $30 \mathrm{kDa}$ [13-15]. As the IgE-reactive bands detected in Golden hamster fur corresponded to the molecular weight expected for MSP, polyclonal rabbit antibodies raised against MSP were used to detect MSP in the different fur extracts (fig. 1a-d, lane R). Major protein bands were detected in the Golden hamster fur extracts at $20 \mathrm{kDa}$ and 24 $\mathrm{kDa}$ as well as a minor band at $30 \mathrm{kDa}$. Similar potentially cross-reactive proteins were recognized at $20 \mathrm{kDa}$ and 24 $\mathrm{kDa}$ in the European hamster fur extracts. No protein bands were detected in the fur extracts of the two dwarf hamsters.

The immunoblots using MSP antibodies suggest that the $20-\mathrm{kDa}$ and $24-\mathrm{kDa}$ IgE-reactive allergens in Golden hamster fur are likely to be MSP lipocalins, and the similar molecular-weight IgE-reactive proteins detected in
European hamster fur are closely related to MSP lipocalins. Overall, the results showed that the IgE-reactive MSP/MSP-like allergens are present in the fur of Golden and European hamsters, and that their epitopes are different from the IgE-reactive 21- to $17-\mathrm{kDa}$ allergens present in the fur of Siberian and Roborovski hamsters.

\section{Immunoblot Inhibition Studies}

In order to confirm that MSP indeed corresponds to the IgE-reactive protein in the fur of the Golden hamster, a series of immunoblot inhibition experiments were carried out on blotted Golden hamster fur proteins (fig. 2a). The binding of IgE antibodies in P4, who had a Golden hamster, was completely inhibited by pre-incubation with purified native MSP proteins, confirming that the 24/20-kDa IgE-reactive proteins share common IgE-reactive epitopes with MSP. However, pre-incubation with Siberian or Roborovski hamster fur extracts did not significantly affect the binding. 
Fig. 2. IgE immunoblot inhibition experiments. Golden, Siberian and Roborovski hamster fur extracts were separated by SDS-PAGE under denaturing conditions and analysed by IgE immmunoblot using 2 patients sera. a Serum of P4, who had a Golden hamster, was preincubated without inhibitor (lane -) or with $20 \mu \mathrm{g}$ of Golden hamster fur extract (lane $G$ ), with $10 \mu \mathrm{g}$ of nMSP (lane M), with $20 \mu \mathrm{g}$ of Siberian (lane S) or Roborovski (lane R) hamster fur extract. b, c Serum of P2, who had a dwarf hamster, was pre-incubated without inhibitor (lane -) or with $20 \mu \mathrm{g}$ of Golden hamster fur extract (lane G), with $20 \mu \mathrm{g}$ of Siberian (lane S) or Roborovski (lane R) hamster fur extract.

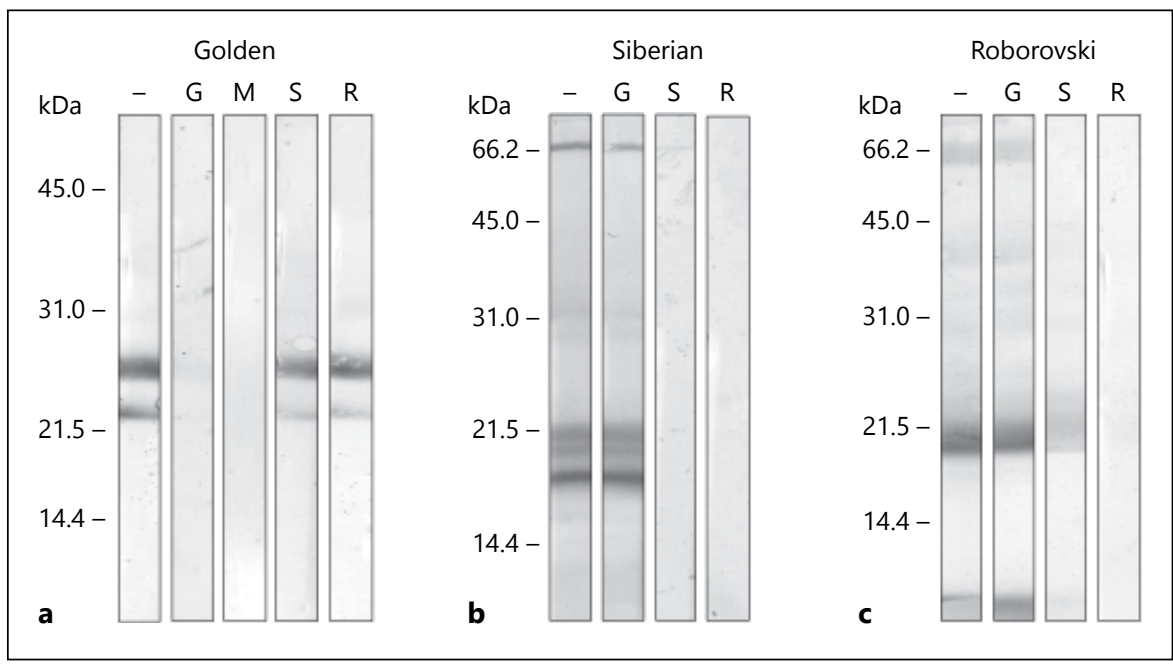

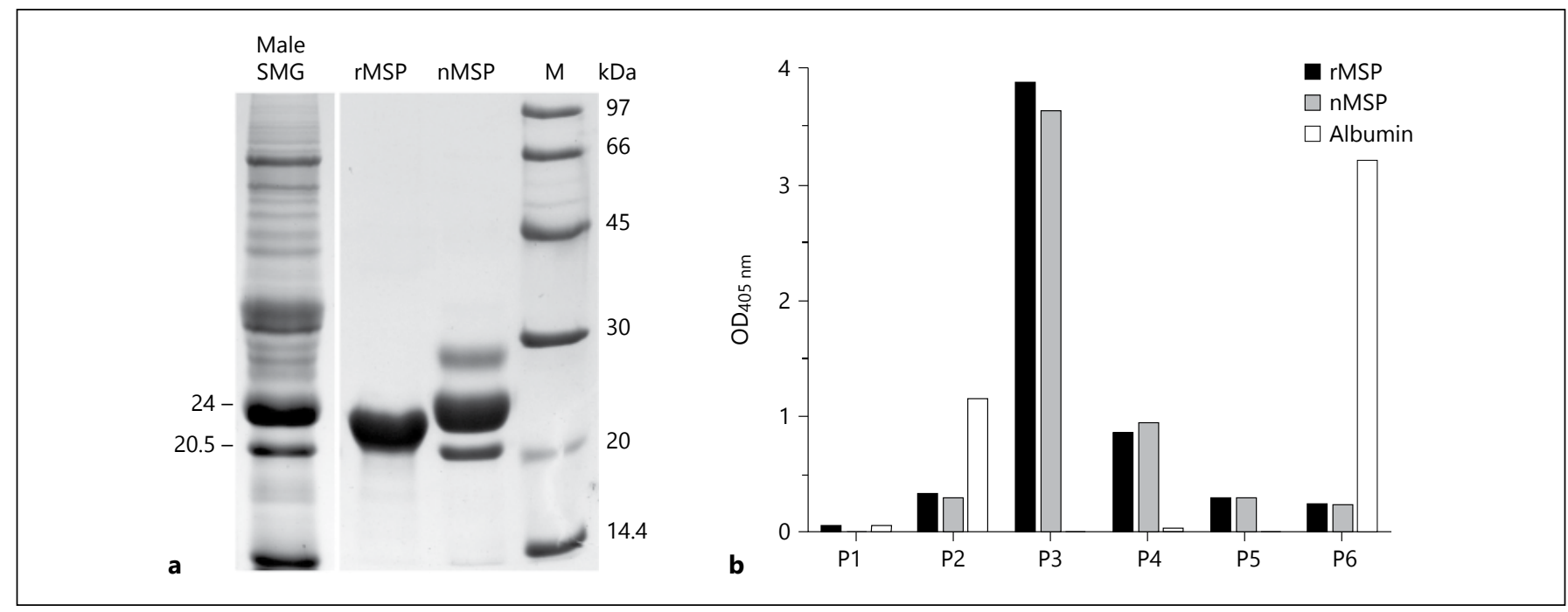

Fig. 3. SDS-PAGE profile of purified MSP proteins and IgE binding to purified hamster proteins by ELISA. a Coomassie-stained SDS-PAGE profile of male Golden hamster SMG extract and purified rMSP and nMSP proteins. Purified nMSP shown is a mixture

IgE-reactivity to fur extracts of Siberian and Roborovski hamsters was further investigated by inhibition and cross-inhibition experiments (fig. 2b, c) using the serum of $\mathrm{P} 2$ who had a dwarf hamster. The reactivity of P2's serum to Siberian hamster fur extract was not affected by pre-incubation with the Golden hamster fur extract, but IgE-binding was completely inhibited by the addition of Siberian or Roborovski hamster fur extract (fig. 2b). In the reverse experiment (fig. 2c), pre-incubation with Siberian hamster fur extract significantly re-

Golden Hamster Allergen MSP Differs from Dwarf Hamster Lipocalin Allergens of the 24-kDa (glycosylated), 20.5-kDa (non-glycosylated) and 30$\mathrm{kDa}$ (glycosylated) forms [13]. b IgE-binding to rMSP, nMSP and serum albumin was assayed by ELISA using the sera of 6 hamsterallergic patients, P1-P6.

duced IgE-binding to the Roborovski extract, but could not completely inhibit it. Complete inhibition was seen after pre-incubation with the Roborovski fur extract. Preincubation with Golden hamster fur extract had no effect.

MSP Is Specifically Recognized by IgE-Antibodies from Hamster-Allergic Patients

All 6 hamster-sensitized patients were tested for IgEreactivity to nMSP, rMSP and hamster serum albumin by ELISA. Figure 3a shows nMSP purified from SMG con- 


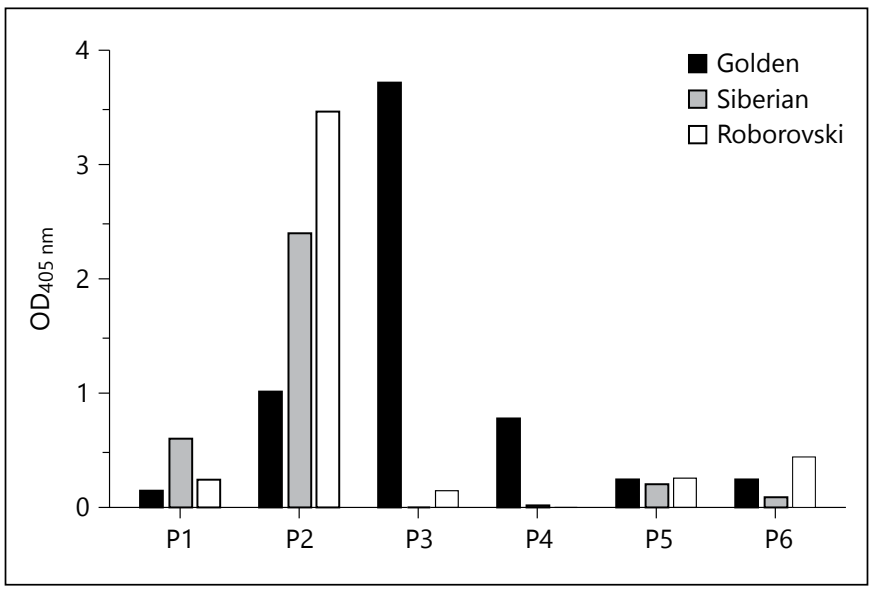

Fig. 4. Detection of IgE reactivity to hamster fur proteins by ELISA. IgE-binding to native proteins extracted from fur of Golden, Siberian and Roborovski hamster was assayed by ELISA using the sera of P1-P6.

taining glycosylated (24 and $30 \mathrm{kDa}$ ) and non-glycosylated (20.5-kDa) forms [13-15] and rMSP purified after expression in E. coli. The sera of all patients except P1 reacted with recombinant and native MSP (fig. 3b). P2 and P6 had also IgE to hamster serum albumin. Twenty-two patients without allergy to animal dander, but with allergies to mite and/or pollen did not show any reactivity to the nMSP or rMSP allergens. In addition, 16 cat- and dogallergic patient sera as well as 10 guinea-pig-allergic patient sera were tested for potential cross-reactivity with MSP, but did not show any IgE-reactivity (data not shown). The MSP lipocalin sequence (GI:19881387) was aligned to known mammalian lipocalin allergens. The most closely related lipocalin allergen was found to be Cav p 3 (42\% identity), followed by Cav p 2 (37\%), while Can f 6, Can f 4, Bos d 2, Equ c 1 and Fel d 4 had 34-30\% identity.

\section{SPT Reactivity to MSP and Fur Extracts from Four Different Hamster Species}

SPTs were performed with the fur extracts of four hamster species: commercial extracts from Golden and European hamster and extracts prepared in-house from the fur of Siberian and Roborovski hamsters (table 1). P1 and P2 reacted strongly with all 4 extracts. P4 who had a Golden hamster reacted only to the Golden and European hamster extracts. She also reacted to nMSP at $0.1 \mu \mathrm{g} / \mathrm{ml}$. P6 reacted strongly to the Golden and European hamster extracts as well as to native MSP at $1 \mu \mathrm{g} / \mathrm{ml}$. The reactivity of P1 to the extracts of the Golden and European hamsters is at variance with the IgE immunoblot experiment. The reactivity of P2 to European hamster fur might be explained by her sensitization to serum albumin, which is present in this extract. Of note, when we determined the protein concentrations of the commercial SPT solutions, they were found to be 30-180 times higher than the concentrations of the in-house extracts which produced a positive reaction.

\section{Detection of IgE Reactivity to Hamster Fur Proteins by ELISA}

The immunoblot experiments analysed patients' reactivity to denatured proteins whereas in the SPTs, patients reacted to native proteins. In order to analyse specific $\operatorname{IgE}$ to native hamster fur proteins, all patient sera were tested by ELISA using protein extracts obtained from the fur of Golden, Siberian and Roborovski hamsters (fig. 4). Sera from P1 and P5, which were negative to Golden hamster fur on immunoblot, showed a weak reaction to Golden hamster fur on ELISA. The serum from $\mathrm{P} 4$ reacted exclusively to Golden hamster fur. This patient did not react in the SPT to the fur extracts of the 2 types of dwarf hamster. The different results obtained by ELISA and immunoblot for P1 and P5 may be explained either by the recognition of structural epitopes, which are denatured in immunoblot and/or by a higher sensitivity in ELISA.

\section{Characterization of IgE-Reactive Proteins from the}

Siberian and Roborovski Dwarf Hamsters

IgE-reactive bands in fur extracts from Siberian and Roborovski hamsters were analysed by N-terminal sequencing. The $21-\mathrm{kDa}$ bands gave the following result: NDYAELEG for the Siberian hamster and NDYAELQGNXDTIA for the Roborovski hamster (X being an unidentified amino acid).

The quality of the obtained sequences was poor due to the low abundance of the proteins in the fur extracts. The MSP allergen, which we found in the fur extracts of the Golden hamster, is known to be abundantly expressed in SMG and is secreted in the saliva [12-15]. We therefore decided to screen the SMGs of Siberian and Roborovski hamsters for the presence of IgE-reactive proteins similar to those detected in their fur extracts. Allergens of molecular weight similar to the ones detected in the fur could be found in the SMGs of both Siberian and Roborovski hamsters (fig. 5a, c). A 2D-gel electrophoresis analysis followed by IgE immunoblot showed that the IgE-reactive bands of both species dissociated into several spots at different acidic isoelectric points (fig. $5 b, d$ ).

These IgE-reactive spots were further analysed by $\mathrm{N}$ terminal sequencing and in-gel trypsin digestion and
36

Int Arch Allergy Immunol 2015;166:30-40 DOI: $10.1159 / 000371420$
Hilger et al. 
Fig. 5. Detection and analysis of IgE-reactive proteins in the SMG extract of Siberian and Roborovski hamsters. SMG proteins $(20 \mu \mathrm{g})$ were separated by SDS-PAGE under denaturing conditions and immunoblotted using the serum of P2. a, b Siberian hamster. c, d Roborovski hamster. $\mathrm{N}=$ Negative control; $\mathrm{P}=$ patient serum. SMG proteins $(250 \mu \mathrm{g})$ were separated by $2 \mathrm{D}$-gel electrophoresis and stained with Coomassie Blue. Arrows and numbers designate the spots analysed by MS/MS.

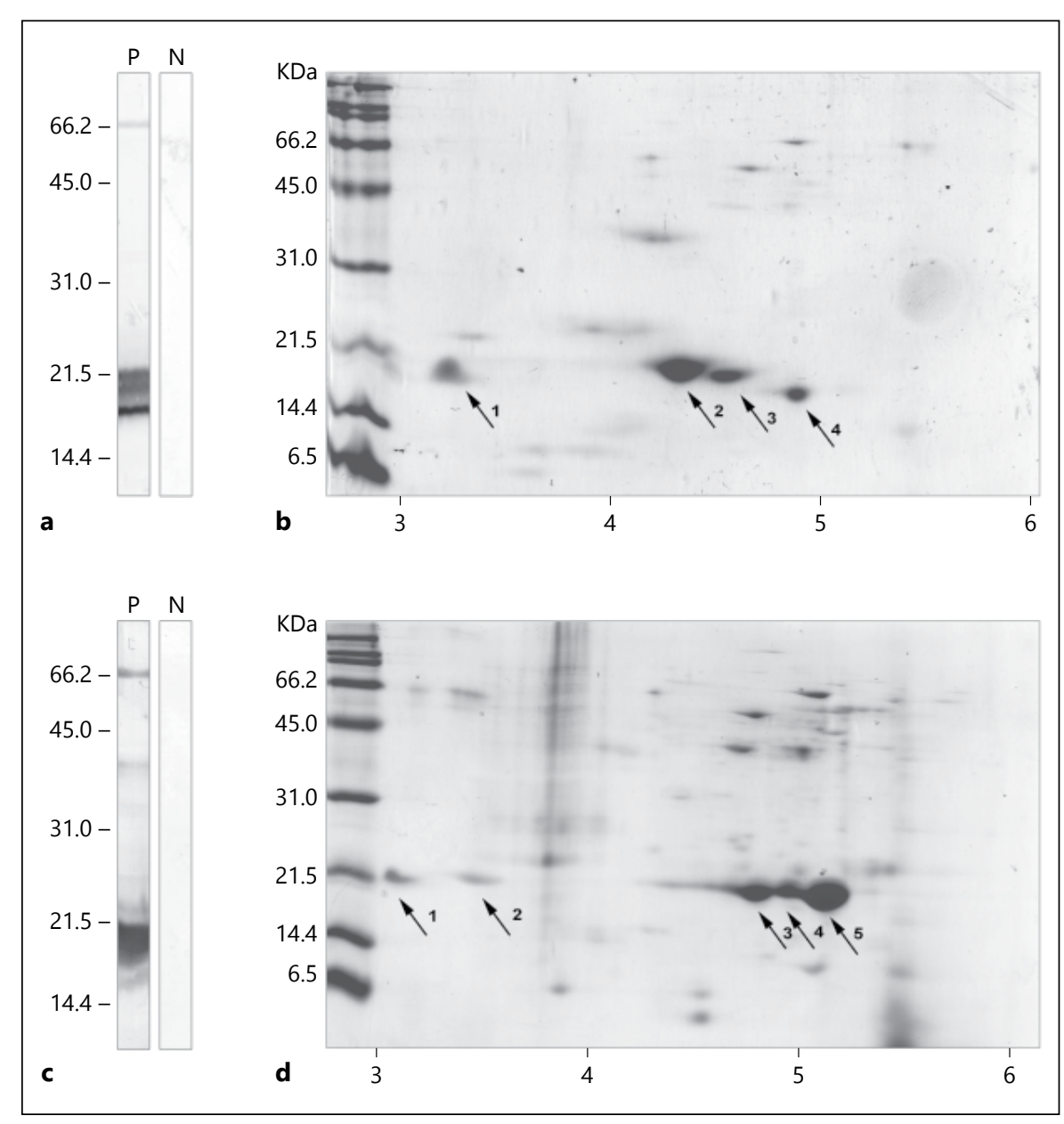

mass spectrometry. Figure 6 shows the amino acid sequences obtained for these spots. No sequence information was obtained for spot 1 (Siberian hamster) as its $\mathrm{N}$ terminal was found to be blocked and it was resistant to enzymatic digestion. The sequence of the N-terminal region obtained for spot $2(21 \mathrm{kDa})$, spot $3(19 \mathrm{kDa})$ and spot $4(17 \mathrm{kDa})$ of the Siberian hamster were identical, except for one mismatching residue at the 26th position $(G)$ in spot 4. Earlier, Torres et al. [7] had sequenced only the 8 amino acids of the N-terminal stretch of 23- and $21-\mathrm{kDa}$ IgE-reactive proteins in the fur, urine and saliva of the Siberian hamster. The sequence (NDYAELEG) obtained by them is completely included in our sequenced $\mathrm{N}$-terminal stretch of spots 2 and 3 of Siberian hamster SMG. The peptide mass fingerprints of the trypsin-digested IgE-reactive spots of the Siberian hamster showed that one of the sequences (spot 2) is identical to the protein sequence deduced from a cDNA of a putative odorant-binding lipocalin (Pho s $21 \mathrm{kDa}$; GI:530376029), which was cloned recently from the Siberian hamster SMG by Torres et al. [19]. Two of the internal peptide sequences determined by Torres et al. [7] are found within the sequence of spot 4 . The few mismatches between the sequences of spots 3 and 4 and their mismatches with that of spot 2 (GI:530376029) indicate the presence of at least 3 isoforms of IgE-reactive allergens in the Siberian hamster.

For the Roborovski hamster, the $36 \mathrm{~N}$-terminal amino acids of the $21-\mathrm{kDa}$ IgE-reactive SMG protein (in $1 \mathrm{D}$ SDS-PAGE) were determined as NDYAELQGN/ KWDTIAIAADNND/AKIKEEGPM/LRLYVREL/H. This N-terminal sequence was highly similar, but not identical to the sequence of roborovskin, a lipocalin protein earlier identified in the urine of Roborovski hamsters, the sequence of which was also determined by Edman degradation and mass spectrometry [11].

Analysis of the IgE-reactive spots obtained by 2D-gel electrophoresis with the Roborovski SMG extract iden- 


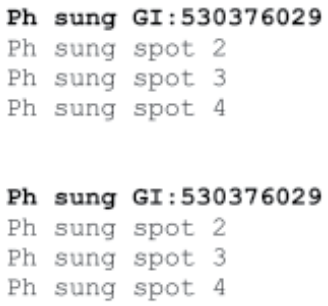

NDYAELEGKWDTIAIAADNDARIKEEGPLRLYVRELYCNEDCSEMEVTFYVNANNQCSKTTVIGYKQADGTYRTQFEGDN 80 NDYAELEGKWDTIAIAADNDAKIKEEGPLR . . . ELYCNEDCSEMEVTFYVNANNQCSKTTVIGYKQADGTYRTQFEGDN

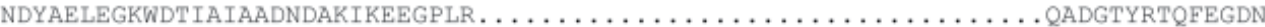

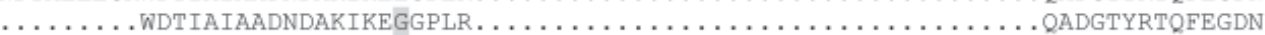

\section{Roborovski hamster}

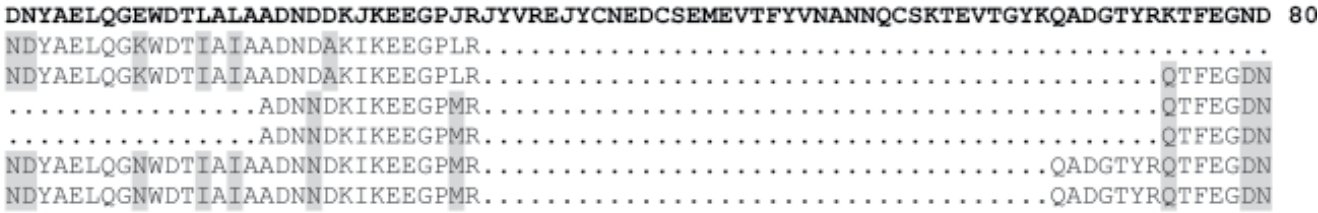

TFKPVYATPEQVVFTSKNVDRAGQETNJJXXXXKSEHJTPEQHEKJVKAJHENNJPEENJHNVJATDTCPK

. FEPVYJTPENJJFTSK . . . . AGQETNJJEVVGK. . HJTPEQHEK . . . . . . . Q JPEENJHNVJATDTCPK

JFQPVYATPENJVFTNR. . . . AGQETNJJFVVGKSQHJSPEQHEK . . . . . . Q JPEENJHNVJATDTCPK JFQPVYATPENJVFTNR $\ldots \ldots \ldots \ldots \ldots \ldots \ldots$ HJTPEQHEK $\ldots \ldots \ldots$ QJPEENJHNVJATDTCPK

JFQPVYATPENJVFTNR $\ldots \ldots \ldots \ldots \ldots \ldots$ SQHJTPEQHEK $\ldots \ldots \ldots$ QJPEENJHNVJATDTCPK

JFQPVYATPENJVFTNRNVDRAGQETNJJFVVGK. .HJTPEQHEK . . . . QJPEENJHNVJATDTCPK

JFQPVYATPENJVFTNRNVDRAGQETNJJFVVGKSQHJTPEQHEK $\ldots \ldots \ldots$ QJPEENJHNVJATDTCPK
Fig. 6. Analysis of protein sequences of IgE-reactive spots isolated from 2D-gel electrophoresis of Siberian and Roborovski hamster SMGs. The protein spots were subjected to Edman sequencing and mass spectrometry analysis. Translated cDNA (GI:530376029) of a Siberian hamster SMG lipocalin and the sequence of roborovskin, a lipocalin isolated from urine of Roborovski hamster [11] were used as references for the mass spectrometry analysis. Isobaric leucine (L) and isoleucine (I) amino acids cannot be distinguished by mass spectrometry and are indicated by $\mathrm{J}$ in the roborovskin and peptide sequences of IgE-reactive spots 3-5, except for in the N-terminal region where they were resolved by Edman degradation. L/I ambiguities within the peptide sequences obtained by mass spectrometry for IgE-reactive spots 2-4 of the Siberian hamster were tentatively resolved by deducting them from the translated cDNA sequence (GI:530376029) of the Siberian hamster. Peptides determined by Torres et al. [7] are underlined. Shaded amino acids indicate the differences between the sequences determined in this study and the reference sequences. $\mathrm{X}=$ Unresolved residue; $d$ ot $=$ no sequence information. tified peptides related to roborovskin and also confirmed the data obtained for the $\mathrm{N}$-terminal sequence of the SMG 21-kDa IgE-reactive band resolved in 1D SDSPAGE. Spots 1 and 2 were resistant to peptide mass fingerprints, but their $\mathrm{N}$-terminal sequences determined by Edman degradation (data not shown) were identical to the $\mathrm{N}$-terminal sequence determined above. The sequence information obtained for spots 3-5 of the IgEreactive $21-\mathrm{kDa}$ SMG protein of the Roborovski hamster indicated the presence of heterogeneity (mismatches among the spots). Thus, several sequence isoforms also exist in the Roborovski hamster, explaining the ambiguous sequence obtained from Edman degradation of the $21-\mathrm{kDa}$ IgE-reactive band of SMG extract and also its heterogenous migration pattern in 2D SDS-PAGE. The isoforms of Roborovski and Siberian hamster allergens do not seem to be glycosylated; they were negative to periodic acid-Schiff stain for glycoprotein (data not shown).

The results of sequencing and mass spectrometry analysis of the IgE-reactive proteins of Siberian and Roborovski hamsters indicated that they are closely related lipocalins with a sequence identity between sequenced stretches of approximately $90 \%$, and they exist as multiple isoforms. In amino acid sequence alignments, Pho s 21 $\mathrm{kDa}$ of the Siberian hamster shows some identity with MSP and Cav p 3 (41\%), followed by Cav p 2 (39\%), Bos d 2 (34\%) and Can f 4, Fel d 4 and Equ c 1 (29-25\%).
38

Int Arch Allergy Immunol 2015;166:30-40 DOI: $10.1159 / 000371420$
Hilger et al. 


\section{Discussion}

Several hamster species are commonly kept as domestic pets. They all belong to the family Cricetinae, but are classified into different genera which form individual clades. The Golden hamster, of the Mesocricetus genus, and the dwarf hamsters of the Phodopus genus separated about 13 million years ago [20]. The $2 \mathrm{~N}$ chromosome numbers of different hamsters vary substantially: the Golden has 44, the European 22, the Siberian 28 and the Roborovski 34 chromosomes [21]. There are other marked differences between these four species, the most prominent being their size, with the European hamster being the largest. It has been described that patients allergic to the Siberian hamster did not react to commercial SPT solutions [2]; such solutions are only available from the Golden and European hamsters.

In this study, we analysed the IgE-reactivity to the fur extracts of four hamster species in order to determine the allergens implicated in allergic reactions to the Golden hamster and define potential cross-reactivity between the allergenic molecules of different hamster species. MSP lipocalin of the Golden hamster, known to be present in the SMG and lacrimal glands, saliva, tears and urine, has now also been detected in body fur. At present, it is unclear whether the MSP in fur is locally synthesized in the glands or cells of the skin or if its presence in the fur is due to contamination by body fluids like saliva and urine which contain MSP [12-15]. Purified native and recombinant MSP were recognized in ELISA by IgE-antibodies in sera from 5 hamster-allergic patients. P4, who was exposed to a Golden hamster, reacted strongly to native MSP in the SPT. MSP seems to be a specific allergen indicative of hamster allergy because it is not recognized by sera from cat-, dog- or guinea-pig-allergic patients. Moreover, MSP-like allergens were also detected in the fur of European hamsters. Sequence identities of MSP to lipocalins of other furry pets are relatively low (max. $42 \%$ ), and a general cross-reactivity seems very unlikely, although the existence of patientdependent cross-reactive epitopes cannot be excluded.

IgE immunoblot inhibition studies were performed using sera of 2 patients, 1 sensitized to the Golden hamster and the other to dwarf hamsters. The results confirmed that MSP is an allergen and that it lacks cross-reactivity with the allergens in the fur of Siberian and Roborovski hamsters. This also confirmed the previous reports of lack of cross-reactivity between allergens of Golden and Siberian hamsters [2, 3, 5, 7]. However, we noted a strong IgE cross-reactivity between the fur allergens of the Siberian and Roborovski hamster.

Golden Hamster Allergen MSP Differs from Dwarf Hamster Lipocalin Allergens
For the Siberian hamster, an allergen had been partially described by Torres et al. [7], who determined the $\mathrm{N}$-terminal sequence for 8 residues as well as 2 short internal peptide sequences for 23- and 21-kDa IgE-reactive proteins, which they detected in the fur, saliva and urine of the Siberian hamster. The $\mathrm{N}$-terminal sequence could not be obtained for an $18-\mathrm{kDa}$ IgE-reactive protein from the same sources, but its internal peptides were identical to those of the $23-\mathrm{kDa}$ and $21-\mathrm{kDa}$ IgE-binding proteins. The authors predicted the existence of 3 isoforms of this allergen, which was classified as a lipocalin based on the sequence information of only 3 peptides. Recently, the same authors cloned a cDNA from the SMG of the Siberian hamster. The encoded lipocalin contains the $\mathrm{N}$ terminal peptide, but shows 4 amino acid deviations from the internal peptides determined previously [7]. The protein, Pho s $21 \mathrm{kDa}$, has been expressed in E. coli, and the binding of IgE-antibodies from 13 patients allergic to Siberian hamster could be demonstrated [19]. Our data confirm the expression of the postulated cDNA at protein level and, based on our extensive sequence data, provide additional evidence for the existence of at least 2 more isoforms, 1 containing the peptides initially described by Torres et al. [7].

The allergens from the Roborovski hamster, another popular pet hamster, were previously unknown. Our analysis of a $21-\mathrm{kDa}$ IgE-reactive protein found in the fur and also abundantly in the SMG of the Roborovski hamster resulted in the identification of this allergen protein as roborovskin, which is a lipocalin, previously reported by Turton et al. [11] in the urine of Roborovski hamsters of both sexes. Our 2D-gel electrophoresis analysis of this IgE-reactive $21-\mathrm{kDa}$ lipocalin allergen showed that it is comprised of several isoforms, resolving as different spots at different acidic isoelectric points. Moreover, we found that the protein sequence of different 2D-gel electrophoresis spots had slight mismatches with each other and also with the sequence of roborovskin obtained in Turton et al. [11]. These differences might be explained by several factors: in their study, Turton et al. [11] purified roborovskin from urine using anion exchange chromatography for MS and MS/MS analysis. Although their source material was different, it is possible that the ion-exchange chromatography resulted in purification of a single isoform and elimination of other variants of roborovskin. Overall, our data show evidence for the existence of at least 3 isoforms of roborovskin, and confirm them to be allergens. Presently, it is unclear whether the observed differences in migration in 2D-gel electrophoresis of the lipocalin allergen isoforms of Siberian and Roborovski

Int Arch Allergy Immunol 2015;166:30-40 DOI: $10.1159 / 000371420$ 
hamsters are only due to protein sequence heterogeneity or also to other post-translational modifications. The different isoforms do not seem to be glycosylated since they were negative to glycoprotein staining.

In conclusion, MSP, a tissue- and sex-specifically expressed lipocalin now found to be also present in body fur, is a major allergen of the Golden hamster. It shares only about $40 \%$ amino acid sequence identity with the major lipocalin allergens of the Siberian and Roborovski hamsters. On the other hand, the amino acid sequences of the lipocalin allergens form Siberian and Roborovski dwarf hamsters are highly identical (approx. 90\%). This finding is in line with clinical observations on a lack of concordance of SPT reactivity of hamster-allergic individuals using commercial extracts. The number of patients analysed in this study being limited, these results have to be confirmed within a larger group of patients with a well-documented clinical history of exposure to different pet hamster species. However, the results pre- sented here as well as in earlier studies clearly establish the need for a SPT formulation using Siberian and Roborovski hamster hair extracts in addition to the existing formulations containing the hair extracts of Golden or European hamster. The specificity of the in vitro IgE diagnosis will also be substantially improved by the isolation and availability of species-specific allergen components in their native or recombinant form.

\section{Acknowledgement}

The project was supported by the Ministry of Higher Education and Research of Luxembourg and by the Department of Science and Technology (India).

\section{Disclosure Statement}

All authors state that they do not have any conflict of interest.

\section{References}

1 Pecquet C: New pets and allergies. Eur J Dermatol 2012;22:14-22.

2 Berto JM, Pelaez A, Fernandez E, Lombardero M, Ferrer M: Siberian hamster: a new indoor source of allergic sensitization and respiratory disease. Allergy 2002;57:155-159.

3 Marenco-Arellano V, Bartolome B, ReanoMartos M, Marrero C, Rodriguez-Cabreros M, Iglesias-Cadarso A: New pets, new allergies. Allergol Immunopathol 2012;40:128-129.

4 Niitsuma T, Tsuji A, Nukaga M, Izawa A, Okita M, Maruoka N, Oguchi A, Morita S, Matsumura $\mathrm{Y}$, Tsuyuguchi M, Hayashi T: Thirty cases of bronchial asthma associated with exposure to pet hamsters. J Investig Allergol Clin Immunol 2004;14:221-224.

5 Lim DL, Chan RM, Wen H, Van Bever HP, Chua KY: Anaphylaxis after hamster bites identification of a novel allergen. Clin Exp Allergy 2004;34:1122-1123.

6 Niitsuma T, Tsuji A, Nukaga M, Izawa A, Okita M, Maruoka N, Morita S, Tsuyuguchi M: Two cases of anaphylaxis after dwarf hamster bites. Allergy 2003;58:1081.

7 Torres JA, Pastor-Vargas C, de las Heras M, Vivanco F, Cuesta J, Sastre J: An odorantbinding protein as a new allergen from Siberian hamster (Phodopus sungorus). Int Arch Allergy Immunol 2012;157:109-112.

8 Gonzalez-Mendiola R, Prieto Montano P, Hinojosa Macias M, Lombardero M, Munoz Martin T: Allergic rhinoconjunctivitis and asthma due to sensitization to Siberian hamster. Allergy 2004;59:1016-1017.
9 Horiguchi T, Tachikawa S, Kasahara J, Kondo R, Miyazaki J, Shiga M, Sugiyama M, Sasaki Y, Hirose M, Teruya S: Clinical studies on bronchial asthma caused by contact with hamsters. Asian Pac J Allergy Immunol 2000;18:141-145.

10 Tomitaka A, Suzuki K, Akamatsu H, Matsunaga K: Anaphylaxis after hamster bites: a rare case? Contact Dermatitis 2002;46:113.

11 Turton MJ, Robertson DH, Smith JR, Hurst JL, Beynon RJ: Roborovskin, a lipocalin in the urine of the Roborovski hamster, Phodopus roborovskii. Chem Senses 2010;35:675-684.

12 De PK: Sex-hormonal regulation of 20.5 and $24 \mathrm{kDa}$ major male-specific proteins in Syrian hamster submandibular gland. J Steroid Biochem Mol Biol 1996;58:183-187.

13 Thavathiru E, Jana NR, De PK: Abundant secretory lipocalins displaying male and lactation-specific expression in adult hamster submandibular gland. cDNA cloning and sex hormone-regulated repression. Eur J Biochem 1999;266:467-476.

14 Srikantan S, Parekh V, De PK: cDNA cloning and regulation of two sex-hormone-repressed hamster tear lipocalins having homology with odorant/pheromone-binding proteins. Biochim Biophys Acta 2005;1729:154-165.

15 Dubey VP, Srikantan S, Mohammad MP, Rajan WD, De PK: Copious urinary excretion of a male Syrian hamster (Mesocricetus auratus) salivary gland protein after its endocrine-like release upon beta-adrenergic stimulation. Gen Comp Endocrinol 2013;186:25-32.
16 Hilger C, Kuehn A, Hentges F: Animal lipocalin allergens. Curr Allergy Asthma Rep 2012;12:438-447.

17 Dubey VP, Pal B, Srikantan S, Pottabathini S, De PK, Sankaranarayanan R: Cloning, overexpression, purification, crystallization and preliminary X-ray analysis of a female-specific lipocalin (FLP) expressed in the lacrimal glands of Syrian hamsters. Acta Crystallogr Sect F Struct Biol Cryst Commun 2010;66:509-512.

18 Prodhomme EJ, Fack F, Revets D, Pirrotte P, Kremer JR, Muller CP: Extensive phosphorylation flanking the C-terminal functional domains of the measles virus nucleoprotein. J Proteome Res 2010;9:5598-5609.

19 Torres JA, de Las Heras M, Maroto AS, Vivanco F, Sastre J, Pastor-Vargas C: Molecular and immunological characterization of the first allergenic lipocalin in hamster: the major allergen from Siberian hamster (Phodopus sungorus). J Biol Chem 2014;289:23382-23388.

20 Hedges SB, Dudley J, Kumar S: TimeTree: a public knowledge-base of divergence times among organisms. Bioinformatics 2006;22: 2971-2972.

21 Romanenko SA, Volobouev VT, Perelman PL, Lebedev VS, Serdukova NA, Trifonov VA, Biltueva LS, Nie W, O’Brien PC, Bulatova N, Ferguson-Smith MA, Yang F, Graphodatsky AS: Karyotype evolution and phylogenetic relationships of hamsters (Cricetidae, Muroidea, Rodentia) inferred from chromosomal painting and banding comparison. Chromosome Res 2007;15:283-297. 